Article

\title{
Computer-Aided Design of Digital Compensators for DC/DC Power Converters
}

\author{
Pablo Zumel *, Cristina Fernández ${ }^{\circledR}$, Marlon A. Granda, Antonio Lázaro and Andrés Barrado \\ Grupo de Sistemas Electrónicos de Potencia, Departamento de Tecnología Electrónica, Escuela Politécnica \\ Superior, Universidad Carlos III de Madrid, 28911 Leganes, Madrid, Spain; cfernand@ing.uc3m.es (C.F.); \\ mgranda@ing.uc3m.es (M.A.G.); alazaro@ing.uc3m.es (A.L.); andres.barrado@uc3m.es (A.B.) \\ * Correspondence: pzumel@ing.uc3m.es; Tel.: +34-916-246-025
}

Received: 4 October 2018; Accepted: 13 November 2018; Published: 22 November 2018

\begin{abstract}
Digital control of high-frequency power converters has been used extensively in recent years, providing flexibility, enhancing integration, and allowing for smart control strategies. The core of standard digital control is the discrete linear compensator, which can be calculated in the frequency domain using well-known methods based on the frequency response requirements (crossover frequency, $f_{c}$, and phase margin, $P M$ ). However, for a given compensator topology, it is not possible to fulfill all combinations of crossover frequency and phase margin, due to the frequency response of the controlled plant and the limitations of the compensator. This paper studies the performance space $\left(f_{c}, P M\right)$ that includes the set of achievable crossover frequencies and phase margin requirements for a combination of converter topology, compensator topology, and sensors, taking into account the effects of digital implementation, such as delays and limit cycling. Regarding limit cycling, two different conditions have been considered, which are related to the design of the digital compensator: a limited compensator integral gain, and a minimum gain margin. This approach can be easily implemented by a computer to speed up the calculations. The performance space provides significant insight into the control design, and can be used to compare compensator designs, select the simplest compensator topology to achieve a given requirement, determine the dynamic limitations of a given configuration, and analyze the effects of delays in the performance of the control loop. Moreover, a figure of merit is proposed to compare the dynamic performance of the different designs. The main goal is to provide a tool that identifies the most suitable compensator design in terms of the dynamic performance, the complexity of the implementation, and the computational resources. The proposed procedure to design the compensator has been validated in the laboratory using an actual DC/DC converter and a digital hardware controller. The tests also validate the theoretical performance space and the most suitable compensator design for a given dynamic specification.
\end{abstract}

Keywords: power converters; digital control; design space; frequency domain

\section{Introduction}

Digital controllers have conquered new areas where analog control was predominant, such as high-frequency DC/DC converters. Features such as special operation modes [1,2], complex control and modulation strategies [3], self-identification [4], autotuning [5-7], communications [8], compensator flexibility, and technology integration [9-11] are the added values provided by digital control.

Digital control of high-frequency converters has been approached using different control techniques. State feedback techniques have been used in [12-14], allowing for the arbitrary placement of the closed loop poles. Full state feedback (inductor current and output voltage) is used in [12] to implement the control law, providing improved performance in a boost converter acting as a 
power factor corrector. In $[13,14]$, state feedback is applied to multiphase power converters to ensure current-sharing. These techniques are suitable for keeping different quantities in control, typically current and voltage. Therefore, they can be considered as an alternative in multiple loop control structures.

Reference [15] proposes a time-domain design method. The digital compensator is derived from specified rise time and overshoot. In [16], the compensator is designed based on the Internal Model Control design method used in motor control. In [17], complex zero compensation is used to improve transient response. In [18], the root locus method is applied to the calculation of a digital controller for a multiphase buck converter.

Frequency domain techniques are very popular when designing analog linear compensators for DC/DC converters [19]. The compensator is calculated by determining the frequency response of the plant and the dynamic requirements regarding their crossover frequency $\left(f_{c}\right)$ and phase margin $(P M)$. A classical design approach, such as the K-factor method [20] ensures that the magnitude of the open-loop gain is $0 \mathrm{~dB}$ at the crossover frequency, and the phase of the open-loop gain provides the required phase margin at that frequency. These design techniques can also be applied to digital linear compensators, considering the discrete nature of the compensator and its implementation, which implies additional factors concerning the analog approach, such as sampling frequency, time delays, and quantization issues. However, the designed controller may not fulfill the expected specifications due to the limitations of the design method itself and the digital implementation-that is, there are $\left(f_{c}, P M\right)$ combinations which are impossible to reach.

In [21], an autotuning system, illustrated with a buck converter, automatically calculated digital PI (proportional-integral), PD (proportional-derivative), and PID (proportional integral derivative) compensators. The $\left(f_{c}, P M\right)$ requirements and the knowledge of the converter dynamics issued from the autotuning system were the basis for the compensator calculation, but this design approach does not consider in detail the attainability of $f_{c}$ and $P M$ requirements. In [22], the digital compensator was calculated directly in the $z$ domain, taking a given value of $f_{c}$ and $P M$ as target performance, without a previous analysis of which of their values could be reached with the proposed compensator type. In [23], the compensator calculation was integrated into the design and optimization process. The design space of the power converter was analyzed considering that the controller was a digital PID, including saturation effects. Different design spaces were proposed, with each one for a particular $\left(f_{c}, P M\right)$ specification.

In all the cited cases, the compensator was calculated from the desired values of $\left(f_{c}, P M\right)$ and the rest of the bandwidth was ignored. Thus, due to the frequency response of the system, the open-loop gain may cross $0 \mathrm{~dB}$ at frequencies different to the crossover frequency, yielding a design that does not meet the dynamic specifications and which could even be unstable. Because of this limitation, each design must be checked after its calculations. If the design does not meet the requirements, it is difficult to decide whether to modify the dynamic specifications, change the topology of the compensator, or modify the sensor.

Thus, it is very interesting and useful for the designer to know the limited set of possible achievable dynamic specifications for a combination of a power converter, compensator, and sensor. This set determines the performance space of the controller regarding the achievable crossover frequency, $f_{c}$, and phase margin, $P M$.

References [24,25] present studies of the design space of analog compensators. However, there are issues related to digital implementation that have an impact on the calculation of the design space and require further investigation, such as sampling frequency, time delays, and limit cycling [26,27]. The consideration of these effects involves additional boundaries and substantially modifies the aspect of the performance space.

This paper proposes a design tool integrating frequency-based design techniques to: (a) determine all the possible solutions without establishing, a priori, the design target $\left(f_{c}, P M\right)$; (b) assess the influence of different parameters of the compensator on the dynamic performance of the system; 
(c) determine the simplest compensator design regarding the dynamic performance for a given dynamic specification, the complexity of the implementation and the computational resources.

This work presents the algorithms to calculate this new performance space and analyze their impact on the controller design. The graphical representation of the performance space in the axis $\left(f_{c}, P M\right)$ can provide the designer with a deep insight on the influence of design parameters through sensitivity analysis. The contribution of this paper regarding prior work is the exhaustive analysis of the digital compensator design (especially in the case of PID); consideration of the limit cycling conditions; and experimental validation.

Section 2 deals with the proposed approach to designing the digital compensator, the description of the limit cycling conditions, and the proposal of a criterion to compare the performance of compensators that provide the same crossover frequency and phase margin. The performance space is introduced in Section 3, defined as a set of feasible and stable designs characterized by their dynamic performance regarding $\left(f_{c}, P M\right)$. Section 4 presents the experimental validation of the proposal. Section 5 summarizes the conclusions of the work.

\section{Elements for the Calculation of the Digital Compensator}

\subsection{Model of the System in the Frequency Domain}

There are different approaches to calculating a digital linear compensator to control a power converter. One of them is based on the calculation of a continuous compensator that fulfills the dynamic requirements [28,29]. This continuous compensator is discretized by means of conventional methods, such as bilinear or Tustin transformation, to obtain the discrete compensator. This approach provides robustness to the design and confidence for analog designers. However, in some cases, the resulting controller is more complex than required because it includes additional poles that are not necessary for the digital controller (e.g., high-frequency poles are not required since the controlled quantity is sampled at the switching frequency).

A second approach is based on calculating the exact model of the converter in the discrete domain, obtaining its $z$ transfer function $[30,31]$. This alternative allows for the direct calculation of the discrete compensator using conventional design techniques for discrete control systems. However, this modeling technique is less extended among the conventional design procedures.

The approach used in this work is based on the frequency response of every block, with its continuous or discrete nature being independent and the frequency range limited to half the switching frequency. Although this is an approximation, it works well, as described in later sections, and provides flexibility to the designer. The elements of the block diagram (Figure 1) are as follows:

- The discrete compensator $C(\omega)$ is the element to be calculated. Its frequency response is calculated by the direct substitution of $z$ by $e^{j \omega \tau_{\text {samp }}}$, where $\tau_{\text {samp }}$ is the sampling period. Despite the sampling period $\tau_{\text {samp }}$ and switching period $\tau$ not necessarily being equal, in this work, they are considered the same for the sake of simplicity $\left(\tau_{\text {samp }}=\tau\right)$;

- Switching power converter $G_{v d}(\omega)$. The more accurate approach to obtaining the frequency response of the power converter (plant) is exact discrete modeling [30,31]. However, good results can also be obtained by using averaged modeling techniques [19,32] or experimental measurements. If a continuous averaged model is used, the small alias approach should be ensured. In the case of exact discrete modeling or experimental results, the effect of a large ripple is inherently considered;

- Time delay $\tau_{\text {delay }}$. All time delays are lumped in a single block despite their origin: modulator [32], analog to digital converter (ADC) and computation. This approximation is valid in most cases. More detailed descriptions could consider the time delay block split in several parts, but this approach is numerically equivalent to the consideration of a single block;

- Static gains of modulator $\left(G_{P W M 0}\right)$ and analog to digital converter $\left(G_{A D C 0}\right)$;

- Sensor transfer function $G_{S}(\omega)$. 
The key transfer function to calculate the compensator is the uncompensated loop transfer function $T_{U}$ given in Equation (1), where $\omega<\pi / \tau$. The open-loop transfer function $T$ is defined in Equation (2).

$$
\begin{gathered}
T_{U}(\omega)=G_{P W M 0} \cdot e^{-j \omega \cdot \tau_{\text {delay }}} G_{A D C 0} G_{v d}(\omega) G_{S}(\omega) \\
T(\omega)=T_{U}(\omega) C(\omega)
\end{gathered}
$$

\subsection{Compensator Calculation}

The calculation of the compensator is based on the specified phase margin and crossover frequency in Equations (3) and (4). Therefore, there are two constraints: the first concerning the definition of the cross-over frequency, $f_{c}$, since the magnitude of the compensated transfer function has to be equal to 1 at $f_{c}$ Equation (3); and the second concerning the definition of phase margin, $P M$ Equation (4).

$$
\begin{gathered}
\left|T_{U}(\omega) \cdot C(\omega)\right|=1=0 \text { dB if } \omega=\omega_{c}=2 \pi \cdot f_{c} \\
\arg \left(T_{U}\left(\omega_{c}\right) \cdot C\left(\omega_{c}\right)\right)=-\pi+P M
\end{gathered}
$$

Without loss of generality, two different compensator types are considered: PI (5) and PID (6).

$$
\begin{gathered}
C_{P I}(z)=K \frac{\left(z-e^{-2 \pi \cdot f_{z} \cdot \tau}\right)}{(z-1)}=K \frac{\left(z-r_{z}\right)}{(z-1)} \\
C_{P I D}(z)=K \frac{\left(z-e^{-2 \pi \cdot f_{z 1} \cdot \tau}\right)\left(z-e^{-2 \pi \cdot f_{z 2} \cdot \tau}\right)}{(z-1) z}=K \frac{\left(z-r_{z 1}\right)\left(z-r_{z 2}\right)}{(z-1) z}
\end{gathered}
$$

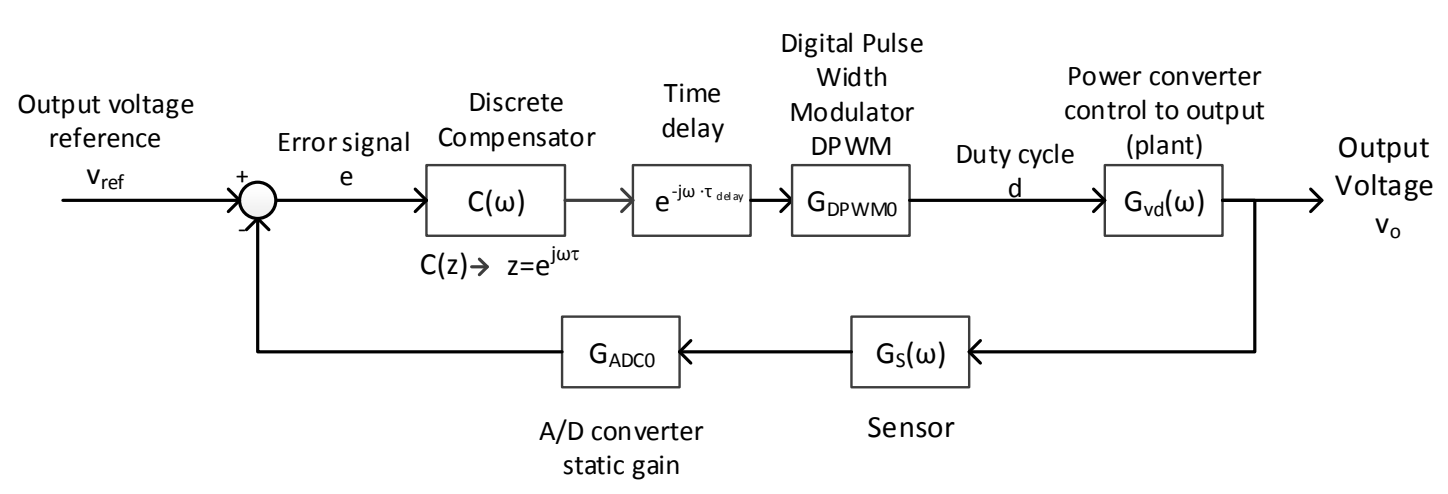

Figure 1. Block diagram of a switched power converter with digital control.

As previously explained, the compensator is calculated from the frequency response of the different blocks of the system. The frequency response of the compensator depends on the topology (PI or PID) and can be calculated by Equations (7) or (8).

$$
\begin{aligned}
C_{P I}(\omega) & =\left.C_{P I}(z)\right|_{z=e^{j \omega \tau}} \\
C_{\mathrm{PID}}(\omega) & =\left.C_{\mathrm{PID}}(z)\right|_{z=e^{j \omega \tau}}
\end{aligned}
$$

In the proposed form, the PI regulator has only two parameters $\left(K\right.$ and $\left.f_{z}\right)$ that can be determined using the constraints (3) and (4). In the case of PI and PID, if the value of $f_{z}$ issued from calculations is negative or complex, the solution must be discarded since it yields zeros outside the unit circle or complex coefficients in the difference equation, respectively.

However, the PID compensator has three parameters $\left(K, f_{z 1}\right.$ and $\left.f_{z 2}\right)$, while there are only two constraints from dynamic requirements $\left(f_{c}\right.$ and $\left.P M\right)$. Therefore, an additional constraint must be 
established to calculate the compensator. In this work, two possibilities are considered as an additional constraint to determine the coefficients of a PID compensator:

- PID1: the ratio between the frequency of one of the zeros $f_{z 2}$ and the crossover frequency $f_{c}$ is given by the designer, as expressed in Equation (9). This approach is a generalization of the proposed calculation criterion given in [33]. In this case, it is possible to derive an analytical expression of the compensator design parameters $K$ and $f_{z 1}$ as a function of the uncompensated transfer function value at $f_{c}, P M$, and $K_{1}$, as depicted in Equations (14)-(16).

$$
\frac{f_{z 2}}{f_{c}}=K_{1}
$$

- PID2: the ratio between the frequency of both zeros is given by the designer, as expressed in Equation (10). When this ratio is equal to one, both zeros are located at the same frequency, and there are analytical expressions to calculate the compensator parameters Equations (17)-(19). However, for other values of $K_{2}$, it is not possible to derive analytical expressions to calculate the parameters directly, because the resulting equation is transcendental. Therefore, numerical methods are required to solve the nonlinear resulting expressions.

$$
\frac{f_{z 2}}{f_{z 1}}=K_{2}
$$

In the following paragraphs, the equations to calculate each of the proposed compensators are summarized. Thus, the expressions for the calculation of proportional-integral (PI) compensator are Equations (11)-(13). Equation (11) is the transfer function, Equation (12) is the difference equation and Equation (13) is the zero location.

$$
\begin{gathered}
C_{P I}(z)=\frac{d(z)}{e(z)}=K \frac{\left(z-r_{z}\right)}{(z-1)}=K \frac{\left(1-r_{z} z^{-1}\right)}{\left(1-z^{-1}\right)} \\
d[n]=d[n-1]+K \cdot e[n]-K \cdot r_{z} \cdot e[n-1] \\
r_{z}=\cos \left(\omega_{c} \tau\right)-\frac{\sin \left(\omega_{c} \tau\right)}{\tan \left(P M-\pi-\arg \left(T_{U}\left(j \omega_{c}\right)\right)+\operatorname{atan}\left(\frac{\sin \left(\omega_{c} \tau\right)}{\cos \left(\omega_{c} \tau\right)-1}\right)\right)}
\end{gathered}
$$

where:

$$
r_{z}=e^{-2 \pi f_{z} \tau} ; K=\frac{1}{\frac{\left|e^{j \omega_{c} \cdot \tau}-e^{-2 \pi f_{z} \tau}\right|}{\left|e^{j \omega_{c} \tau}-1\right|}\left|T_{U}\left(\omega_{c}\right)\right|}
$$

Expressions for the calculation of proportional-integral-derivative PID1 compensator are Equations (14)-(16). The transfer function is Equation (14), the difference equation is Equation (15) and Equation (16) provides the location of zero 1 (note that the frequency of the other zero is determined by the value of $K_{1}$ ).

$$
\begin{gathered}
C_{P I D 1}(z)=\frac{d(z)}{e(z)}=K \frac{\left(z-r_{z 1}\right)\left(z-r_{z 2}\right)}{(z-1) z}=K \frac{\left(1-r_{z 1} z^{-1}\right)\left(1-r_{z 2} z^{-1}\right)}{\left(1-z^{-1}\right)} \\
d[n]=d[n-1]+K \cdot e[n]-K\left(r_{z 1}+r_{z 2}\right) \cdot e[n-1]+K\left(r_{z 1} \cdot r_{z 2}\right) \cdot e[n-2] \\
r_{z 1}=\frac{B \cdot D-A+(C-B \cdot E) \cdot K_{z}}{\left(B \cdot E-C-B \cdot K_{z}\right)}
\end{gathered}
$$

where:

$$
A=\sin \left(2 \omega_{\mathcal{c}} \tau\right) ; B=\tan \left(P M-\pi+\arg \left(e^{j \omega_{\mathcal{c}} \tau}-1\right)+\omega_{\mathcal{c}} \tau-\arg \left(T_{U}\left(\omega_{\mathcal{c}}\right)\right)\right) ; C=\sin \left(\omega_{\mathcal{c}} \tau\right) ;
$$




$$
\begin{gathered}
D=\cos \left(2 \omega_{\mathcal{C}} \tau\right) ; E=\cos \left(\omega_{\mathcal{c}} \tau\right) ; \quad K_{z}=e^{-\omega_{c} K_{1} \tau} ; r_{z 1}=e^{-2 \pi f_{z 1} \tau} ; r_{z 2}=e^{-2 \pi f_{z 2} \tau} \\
K=\frac{1}{\frac{\left|e^{j \omega_{c} \cdot \tau}-e^{-2 \pi f_{z 1} \tau}\right| \cdot\left|e^{j \omega_{c} \cdot \tau}-e^{-2 \pi f_{z 2} \tau}\right|}{\left|e^{j \omega_{\mathcal{C}} \tau}-1\right| \cdot\left|e^{\omega_{\mathcal{c}} \tau}\right|}\left|T_{U}\left(\omega_{\mathcal{C}}\right)\right|}
\end{gathered}
$$

Finally, expressions for the calculation of PID2 compensator are Equations (17)-(19). The transfer function if Equation (17), the difference equation is Equation (18). Equation (19) is applicable when both zeros are located at the same frequency $\left(K_{2}=1\right)$.

$$
\begin{gathered}
C_{P I D 2}(z)=\frac{d(z)}{e(z)}=K \frac{\left(z-r_{z 1}\right)\left(z-r_{z 2}\right)}{(z-1) z}=K \frac{\left(1-r_{z 1} z^{-1}\right)\left(1-r_{z 2} z^{-1}\right)}{\left(1-z^{-1}\right)} \\
d[n]=d[n-1]+K \cdot e[n]-K\left(r_{z 1}+r_{z 2}\right) \cdot e[n-1]+K\left(r_{z 1} \cdot r_{z 2}\right) \cdot e[n-2] \\
r_{z}=\cos \left(\omega_{\mathcal{c}} \tau\right)-\frac{\sin \left(\omega_{\mathcal{c}} \tau\right)}{\tan \left(\frac{1}{2}\left(P M-\pi-\arg \left(T_{U}\left(j \omega_{\mathcal{c}}\right)\right)+\operatorname{atan}\left(\frac{\sin \left(\omega_{\mathcal{c}} \tau\right)}{\cos \left(\omega_{\mathcal{c}} \tau\right)-1}\right)+\omega_{\mathcal{c}} \tau\right)\right)}
\end{gathered}
$$

where:

$$
r_{z}=r_{z 1}=r_{z 2}=e^{-2 \pi f_{z} \tau} ; \quad K=\frac{1}{\frac{\left|e^{j \omega_{c} \cdot \tau}-e^{-2 \pi f_{z} \tau}\right|^{2}}{\left|e^{j \omega_{\mathcal{C}} \tau}-1\right| \cdot\left|e^{j \omega_{c} \tau}\right|}\left|T_{U}\left(\omega_{\mathcal{C}}\right)\right|}
$$

In the case of PID2 compensator with two zeros at different frequency $\left(r_{z 1} \neq r_{z 2}\right)$, a numerical approach is used to find the frequency of the zeroes.

\subsection{Analysis of the Calculated Compensators}

The expressions (11)-(19) provide the compensator parameters to meet the requirements of gain and phase only at the crossover frequency. However, as mentioned in the introduction, because of the frequency response of the plant, the behavior of the open-loop gain at other frequencies can render the solution unstable or not feasible, or even result in a different dynamic performance than expected. Thus, it is interesting to analyze these possibilities considering the whole bandwidth of the loop transfer function.

Five combinations of crossover frequency and phase margin have been considered to analyze the different possibilities. The resulting linear compensators yield the open-loop gain transfer functions illustrated in Figure 2. The plant considered in the calculations is a buck converter (input voltage Vin $=12 \mathrm{~V}$, output voltage $\mathrm{Vo}=3 \mathrm{~V}$; output inductance $\mathrm{L}_{\mathrm{O}}=1 \times 10^{-6} \mathrm{H}$; output capacitance $\mathrm{C}_{\mathrm{O}}=47 \times 10^{-6} \mathrm{~F}$; output power $\mathrm{Po}=10 \mathrm{~W}$; switching frequency fsw $=1000 \times 10^{3} \mathrm{~Hz}$; total time delay $\mathrm{td}=(1 / \mathrm{fsw}) \times 0.5)$. In the plots, the continuous line represents the theoretical frequency response, while the crosses correspond to the frequency response obtained from time-domain simulations using the discrete compensator performing and ACsweep with the commercial simulator PSIM.

Case 1 (target $f_{c}=84 \mathrm{kHz}$, target $P M=45^{\circ}$ ) is a feasible solution, since the magnitude of the open-loop gain crosses $0 \mathrm{~dB}$ only once, the phase crosses $-180^{\circ}$ only when the magnitude is lower than $0 \mathrm{~dB}$, and the obtained zero frequency is real and positive.

Case 2 (target $f_{c}=79 \mathrm{kHz}$, target $P M=15^{\circ}$ ) is a conditionally stable solution, because the phase is lower than $-180^{\circ}$ at frequencies where the magnitude of the open-loop gain is higher than $0 \mathrm{~dB}$. The point -1 is outside the Nyquist contour, but a decrease in the gain of the system could make the system unstable. Thus, this solution is discarded despite the resulting system not being formally unstable, and the crossover frequency and phase margin should not be included inside the performance space.

Case 3 (target $f_{c}=20 \mathrm{kHz}$, target $P M=75^{\circ}$ ) is unstable because the phase is lower than $-180^{\circ}$ when the magnitude is higher than $0 \mathrm{~dB}$. Therefore, this solution must be discarded. 

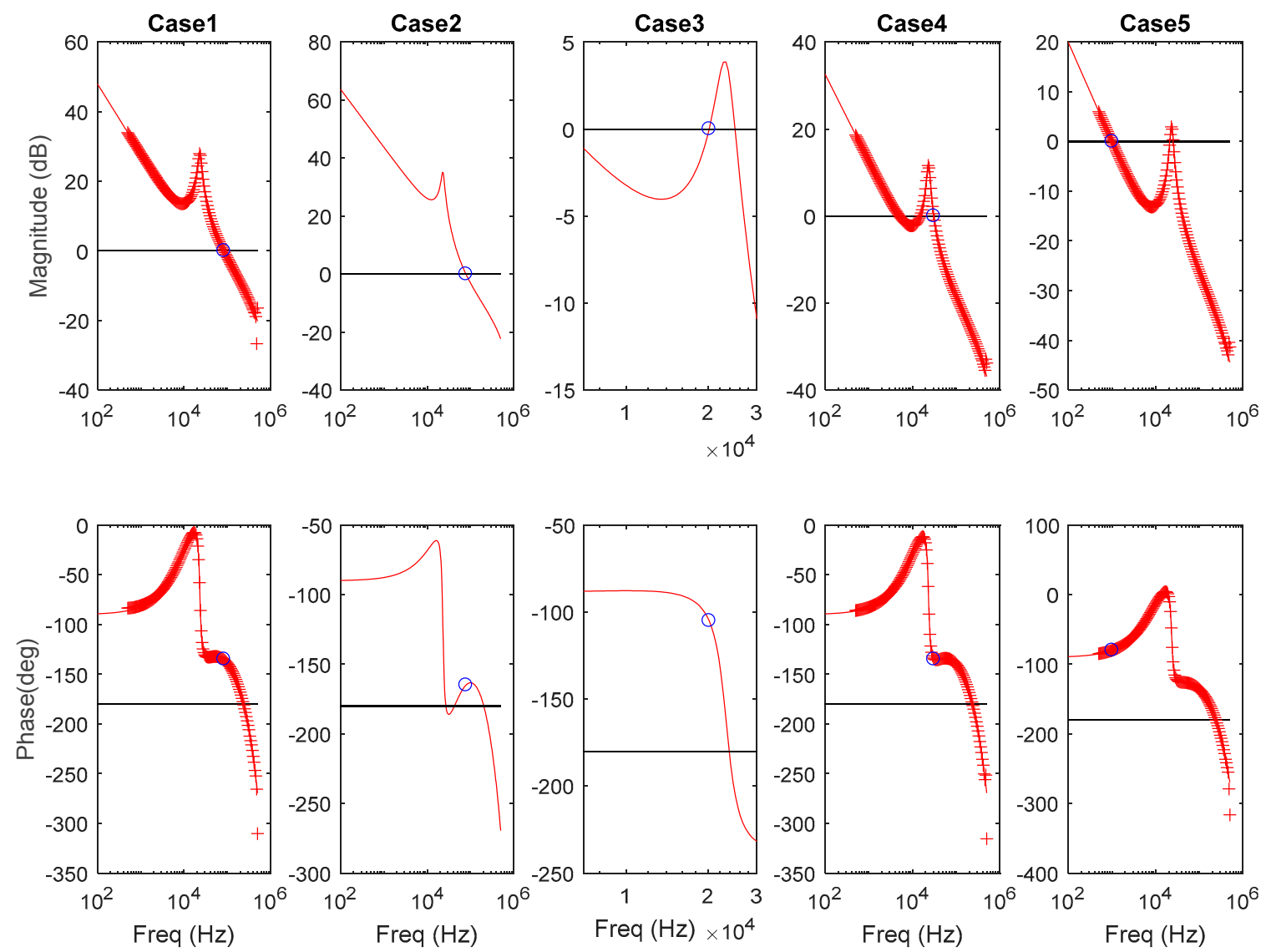

Figure 2. Open-loop transfer function for different combinations of crossover frequency and phase margin. Solid line: theoretical freq. response; crossed line: freq. response from the time-domain simulation with a PSIM simulator.

Case 4 (target $f_{c}=20 \mathrm{kHz}$, target $P M=75^{\circ}$ ) is stable, but the effective crossover frequency is lower than expected due to the two crossings through $0 \mathrm{~dB}$ that appear approximately at $5 \mathrm{kHz}$ and $15 \mathrm{kHz}$. The result is that the settling time of the system does not correspond with the target crossover frequency, and the system is slower than expected. Thus, with this design procedure and digital compensator, it is not possible to achieve the specified combination of crossover frequency and phase margin. This combination should be considered out of the performance space.

Case 5 (target $f_{c}=1 \mathrm{kHz}$, target $P M=100^{\circ}$ ) is stable, but the effective crossover frequency is higher than expected. This means that the dynamic response of the system does not match the requirements. Moreover, the effective phase margin is lower. Therefore, this solution is also discarded.

The main conclusion of this analysis is that there are combinations of dynamic specifications that yield designs which should be considered out of the performance space. Reasons to discard the resulting designs are as follows:

- The magnitude of the resulting open-loop gain crosses $0 \mathrm{~dB}$ more than once: the solution is discarded since the effective phase margin is different than expected. Thus, the resulting design does not fulfill the dynamic specifications;

- The phase of the resulting open-loop gain crosses $-180^{\circ}$ with a magnitude greater than $0 \mathrm{~dB}$ : the solution is discarded because it is unstable or conditionally stable.

\subsection{Limit Cycling Conditions}

One of the characteristics of digitally controlled power converters is the limit cycles that can appear under different conditions. This issue is still a subject of research, especially in the case of 
transient conditions; but in the literature, different conditions have been established to avoid limit cycling [26]. Some of them are related to the relative resolution between the PWM (pulse width modulator) and the ADC. These are out of the scope of this work, since they do not depend on the compensator calculation, but rather their hardware implementation. However, there are other two conditions related to the design of the linear compensator.

The first condition [26,27] is related to the compensator integral gain $K_{i}$, defined in Equation (20). The expressions of the integral gain $K_{i}$ for PI and PID compensators are given in Equations (21) and (22) respectively.

$$
\begin{gathered}
K_{i}=\lim _{z \rightarrow 1}(z-1) C(z) \\
K_{i}=K \cdot\left(1-r_{z}\right) \\
K_{i}=K\left(1-r_{z 1}\right)\left(1-r_{z 2}\right)
\end{gathered}
$$

This integral gain $K_{i}$ must be limited in order for a single unit impulse of the error signal to produce a step change in the controlled quantity (e.g., output voltage). This condition is expressed in Equation (23). Theoretically, the parameter $a$ is equal to 1 , but in practice a, safety factor is considered, which can typically be $a=0.5$.

$$
0<T_{U}(\omega=0) \cdot K_{i}<a
$$

Once the digital compensator is calculated, it is easy to check if it meets Equation (23) to determine whether the solution should be discarded and considered out of the performance space. However, when analyzing Equations (21) and (22), interesting trends can be identified. In a PI compensator, as $K$ and $r_{z}$ are directly determined by the constraints imposed by $f_{c}$ and $P M$, the only possibility to avoid limit cycling is to change $\left(f_{c}, P M\right)$; that is, to modify the dynamic requirements. On the other hand, in the case of a PID compensator (topologies PID1 and PID2), the additional degree of freedom can be used to avoid limit cycling without changing the $f_{c}$ and $P M$ requirements, but by changing the parameters of $K_{1}$ or $K_{2}$ in Equations (9) and (10).

This effect is illustrated in Figure 3. The plots show the value of $T_{U}(\omega=0) \cdot K_{i}$ depending on the parameters $K_{1}$ or $K_{2}$ and for a given combination of $f_{c}$ and $P M$. When $K_{1}$ or $K_{2}$ are small enough, the plotted product is lower than $a=1$, so a limit cycling condition is avoided. The conclusion is that by decreasing the frequency of one of the PID zeros, the gain of the system at low frequency is limited enough to avoid the integral gain limit cycling. However, one zero at low frequency can result in a poor low frequency response, as will be shown in the next section. Therefore, a trade-off must be found.

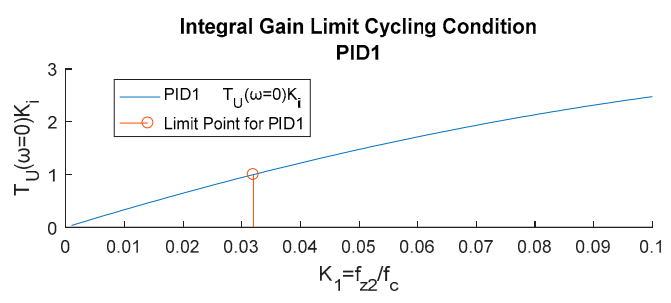

(a)

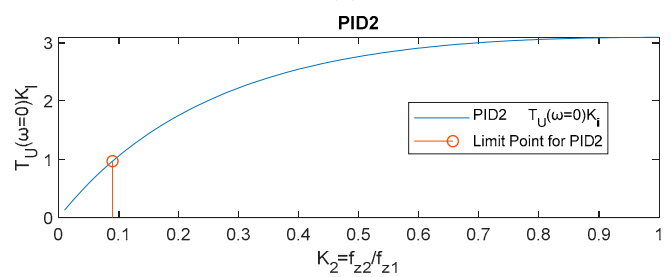

(b)

Figure 3. Analysis of the integral gain limit cycling condition for the PID1 (a) and PID2 (b) compensators. 
The second limit cycling condition refers to the requirement of a minimum gain margin, $G M$, to avoid limit cycling due to the sampling effect in Equation (24), where the parameter $\alpha$ is the security margin.

$$
G M>4.2 \mathrm{~dB}-20 \cdot \log (\alpha)
$$

This condition depends essentially on the crossover frequency $f_{c}$. When a high $f_{c}$ is desired, close to the Nyquist frequency, the gain margin is limited, since the slope of $T$ is limited for a PID compensator beyond $f_{c}$. Thus, changing the value of the PID parameter has a very slight effect on the $G M$, since beyond $f_{c}$, the value of $T$ is very similar even if there are significant differences for low frequencies, as illustrated in the open-loop gain displayed in Figure 4a.

\subsection{Difference among Solutions with the Same Crossover Frequency and Phase Margin}

According to the previous section, for a given $f_{c}$ and $P M$, there is only one possible design if all the parameters are fixed: the type of compensator, sampling frequency, parameters $K_{1}$ or $K_{2}$, and so forth. Following the previously described criteria, it is possible to determine which designs are included in the performance space and which are not. However, it would be interesting to provide a method to compare the different solutions and indicate the best one in terms of the complexity of the implementation, the computational resources, and so forth. Thus, a PI compensator is preferred to a PID for the same crossover frequency and phase margin, since the difference equation has fewer coefficients (expressions (11)-(19)). The compensator with a lower sampling period is preferred, since its implementation needs fewer digital resources.

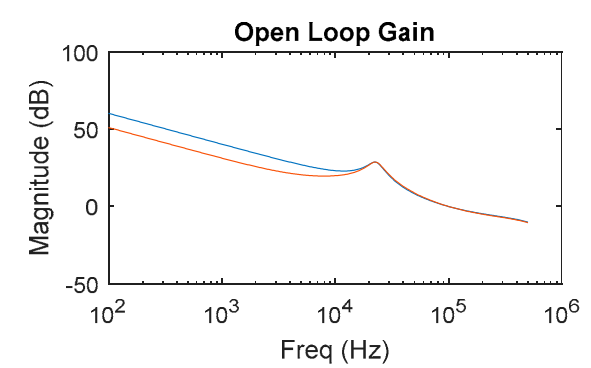

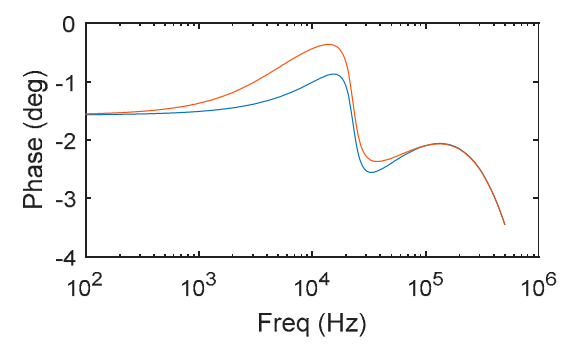

(a)

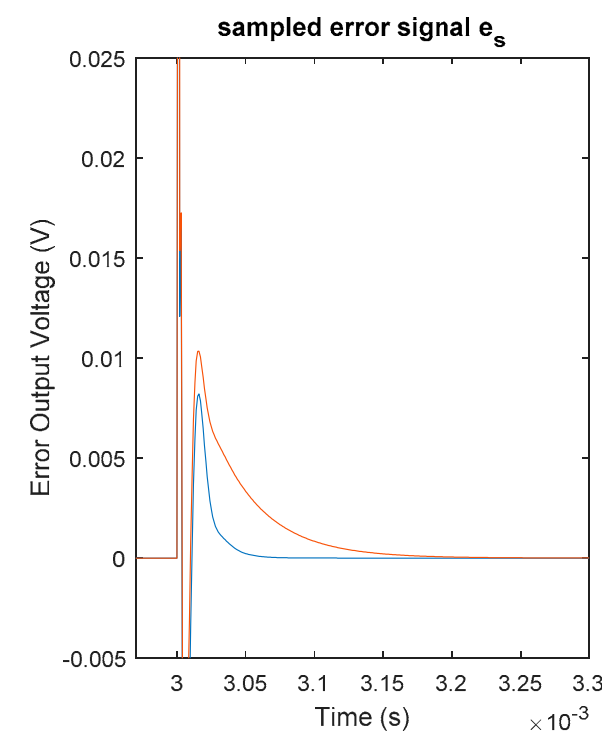

(b)

Figure 4. (a) Open-loop gain T, corresponding to two different designs of PID2 with the same $\left(f_{c}, P M\right)$ and different parameter values of $K_{2} ;(\mathbf{b})$ error signal in the time domain when a step in the reference voltage is applied.

However, for the PID compensator, there are different possible designs, since an additional design constraint is used either in PID1 and PID2. Therefore, a performance index should be established to choose between compensators with the same architecture and different parameters.

Figure 4 illustrates this issue. Two different PID2 compensators with different values of $K_{2}$ have been calculated. Both achieve the same $f_{c}$ and $P M$, but their frequency response is different in the rest of the bandwidth (Figure 4a). The difference in magnitude is approximately $9 \mathrm{~dB}$ in the range of $100-1000 \mathrm{~Hz}$ for this illustration example. Thus, the transient response for a reference step is also different. When evaluating the error output voltage signal in the time domain - that is, the difference 
between the output voltage reference (reference signal) and the actual output voltage (measured controlled signal) in Figure $4 \mathrm{~b}$, the design with a lower magnitude in the range of 100-1000 Hz exhibits a longer settling time.

To compare different designs with the same $f_{c}$ and $P M$ as those appearing in Figure 4, the performance index $L$ is defined in Equation (25). This index is the norm of the difference between the closed loop transfer function of the system $C L\left(f_{k}\right)$ compared with the ideal one, which is equal to 1 at any frequency; $f_{k}$ is the $k$-th element of the frequency vector, considering that the proposed design approach is numerical. $C L\left(f_{k}\right)$ is obtained from the open-loop transfer function $T\left(f_{k}\right)$. A weighting factor $\frac{1}{f_{k}}$ is included to give more importance to the low frequency response. The factor $\frac{f_{k}-f_{k-1}}{f_{\max }-f_{\min }}$ is considered to take the non-linear spacing in the frequency vector into account.

$$
L=\sqrt{\sum_{k=1}^{N}\left|\left(C L\left(f_{k}\right)-1\right) \cdot \frac{1}{f_{k}}\right|^{2} \cdot \frac{f_{k}-f_{k-1}}{f_{\text {max }}-f_{\text {min }}}}=\sqrt{\sum_{k=1}^{N}\left|\left(\frac{T\left(f_{k}\right)}{1+T\left(f_{k}\right)}-1\right) \cdot \frac{1}{f_{k}}\right|^{2} \cdot \frac{f_{k}-f_{k-1}}{f_{\text {max }}-f_{\text {min }}}}
$$

The ideal value of this index is $L=0$, which means that the closed-loop transfer function is equal to 1. Comparing two different solutions-that is, two different compensators, the best solution corresponds to the minimum value of index $L$. It is difficult to find an exact relationship between the proposed index $L$ and the conventional time domain indexes. However, in all tested cases, a higher value of $L$ means a higher error in the time domain, as illustrated in Figure 5, where six different PID designs for the same $\left(f_{c}, P M\right)$ are compared. The parameters changing from one design to a different one are $K_{1}$ and $K_{2}$, as defined in Equations (9) and (10) respectively - that is, the separation between zeros of the compensator. Designs A have higher values of $K_{1}$ and $K_{2}$ than designs B, and both have higher values of $K_{1}$ and $K_{2}$ than designs $C$. In Figure $5 a$, the frequency response for the different designs is shown. In this particular case, designs PID2B and PID1B are very similar (for example, PID2B is $0.08 \mathrm{~dB}$ greater than PID1B at $400 \mathrm{~Hz}$ ). In Figure 5b, the RMS (root mean square) value of the error signal in the time domain is plotted for the six designs, considering an output voltage reference step and normalizing the values to the lowest RMS value. In Figure $5 c$, the value of the proposed $L$ index normalizing the value to the lower $L$ index value is shown. Although there is no linear relationship between the time-domain error RMS value and $L$ index value, the lower the $L$ index value, the lower the error RMS value.

Time-response performance indexes are an extended tool to compare compensator performance [34]. However, since the presented design approach is based on the frequency domain, it is desirable to establish a performance index based on the frequency response to quickly compare different compensator performances. Note that if the design method requires the comparison of many solutions, saving computational resource is important. 

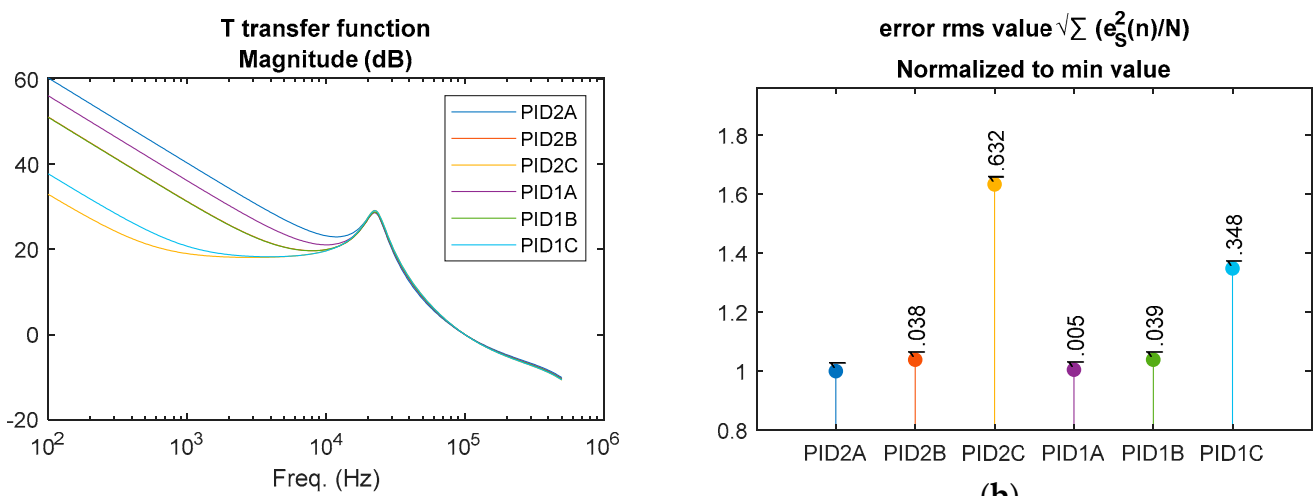

$T$ transfer function

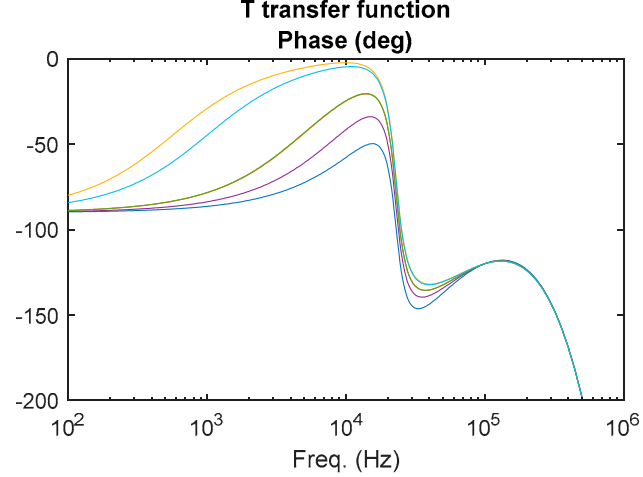

(a) (b)

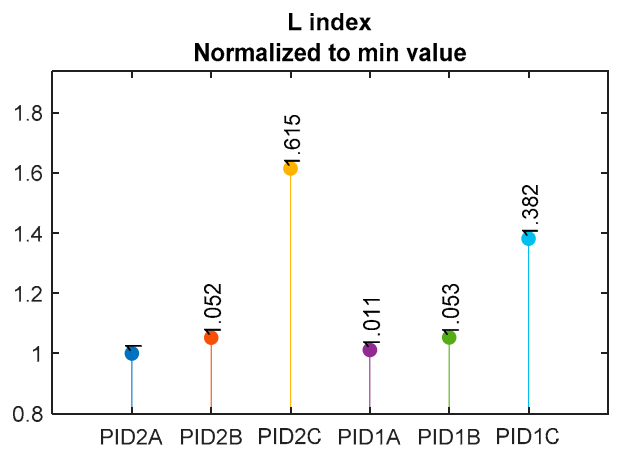

(c)

Figure 5. Comparison of solutions with the same $\left(f_{c}, P M\right)$ pair corresponding to different compensator types and parameters. (a) Open-loop transfer function; (b) RMS value of the error signal obtained from the simulation; (c) $L$ index obtained from the open-loop transfer function.

\section{Determination of the Performance Space $\left(f_{c}, P M\right)$}

The previous section introduced the frequency-based model of the system, the calculation of different compensator topologies, the criteria to determine whether the designs fulfill the dynamic requirements, and an index to compare compensators that provide the same dynamic specifications $\left(f_{c}, P M\right)$.

However, significant advantages can be obtained if the proposed calculation procedure is automated and applied to find a performance space in such a way that all the possible combinations of $\left(f_{c}, P M\right)$ that can be fulfilled are found. Thus, the performance space is graphically represented in a plot with frequency units in the horizontal axis and phase units in the vertical axis. In [25], this plot is called a solution map. In this paper, the design space is the set of parameters of the compensator that have to be calculated to obtain given results regarding $\left(f_{c}, P M\right)$ - that is, to obtain a given performance. Those parameters of the compensator are, finally, the coefficients of the difference Equations (12), (15) and (18), and the performance is the pair $\left(f_{c}, P M\right)$. In the following paragraphs, an example illustrates the generation of the performance space $\left(f_{c}, P M\right)$ and the influence of different factors, such as the type of compensator and limit cycling conditions. The specifications of the power converter used as our example are as follows: buck topology, input voltage Vin $=12 \mathrm{~V}$, output voltage Vo $=3$; output inductance $\mathrm{L}_{\mathrm{O}}=1 \times 10^{-6} \mathrm{H}$; output capacitance $\mathrm{C}_{\mathrm{O}}=47 \times 10^{-6} \mathrm{~F}$; capacitor equivalent series resistance $\mathrm{R}_{\mathrm{ESRC}}=20 \times 10^{-3} \Omega$; output power $\mathrm{Po}=10 \mathrm{~W}$; switching frequency fsw $=1000 \times 10^{3} \mathrm{~Hz}$; total time delay $\mathrm{td}=(1 / \mathrm{fsw}) \times 0.5$.

In Figure 6a, the uncompensated open-loop transfer function $T_{u}$ defined in Equation (1) is shown, including time delays, static gains, and the converter-transfer function (Figure 1). In Figure $6 b$, the described performance space for the example buck converter and a PID1 compensator is plotted. Limit cycling conditions are not involved in these first calculations. Every point represents a combination 
of $f_{c}$ and $P M$, and the valid combinations are grouped into different areas, considering the different possibilities discussed in Section 2.3:

- The white area corresponds to solutions with non-real or negative frequencies;

- The pink area is the set of solutions which are unstable or conditionally stable. This area is particularly large at high frequencies;

- The blue area is the set of valid solutions that corresponds to feasible stable designs with the same $f_{c}$ and $P M$ as plotted in the performance space.

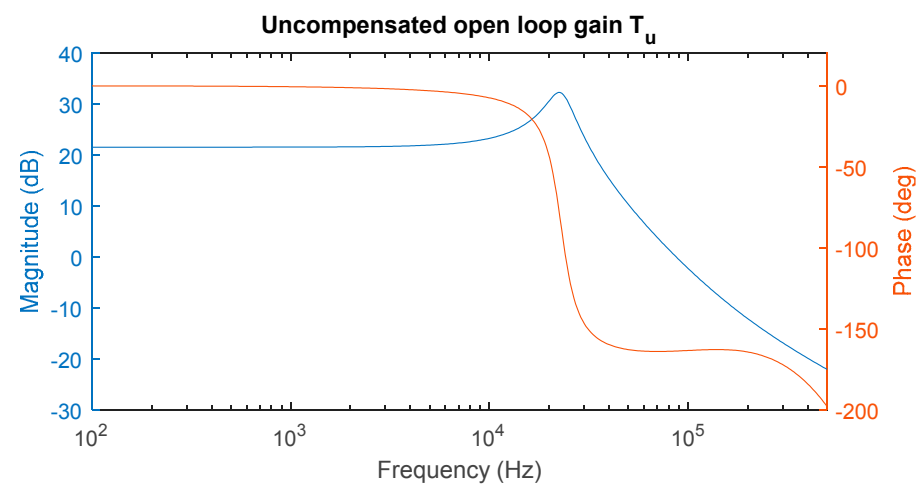

(a)

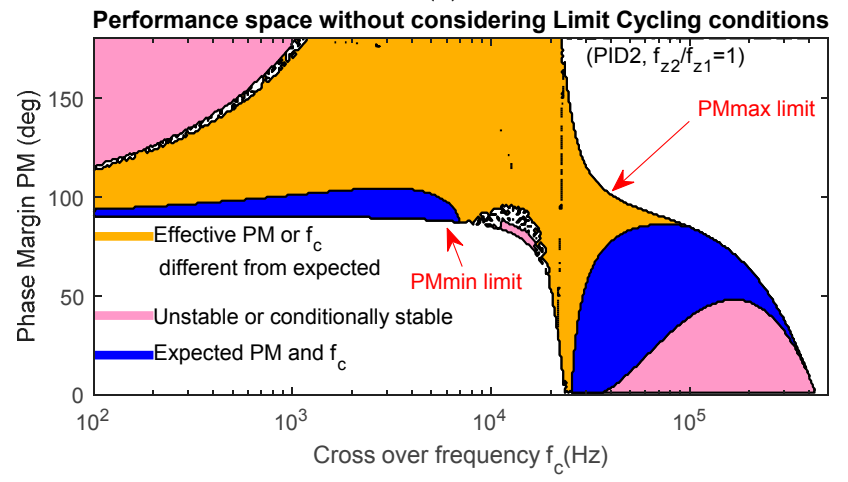

(b)

Figure 6. (a) Bode plot of the uncompensated transfer function, $\mathrm{Tu}$; (b) performance space for a PID1 compensator, without considering limit cycling conditions.

The chosen plant, in this case, exhibits a relatively high $Q$ - that is, the resonance peak is around $10 \mathrm{~dB}$ above the low frequency magnitude of the $G_{v d}$ transfer function. This fact is relevant to producing pairs $\left(f_{c}, P M\right)$ corresponding to cases 2, 3, 4, and 5 in Figure 2.

The algorithm to calculate the performance space is described in the flowchart of Figure 7. It has been elaborated using the theoretical basis provided in Section 2. The maximum PM that can be achieved (PMmax limit in Figure 6b) can be theoretically calculated as the phase of the uncompensated open-loop transfer function $T_{U}$, plus the maximum phase boost that the compensator can provide. On the other hand, there is a limit at low frequencies for the PM (PMmin limit in Figure 6b), determined by the phase of the uncompensated open-loop transfer function $T_{U}$ and the minimum phase provided by the compensator ( -90 degree in any case). These two boundaries are relevant to reduce the calculations and shorten the calculation time of the performance space [35]. Once the initial limits of PM have been established, a double sweep is carried out. For each frequency, the value of PM is initialized and the validity conditions are checked, including those described in Section 2.2, 0 and 0 . If they are met, the value of $P M$ is increased, and the validity conditions are checked again. 
Once a non-valid solution is achieved for a given frequency value, the PM loop is interrupted, and the algorithm goes to the next frequency value.

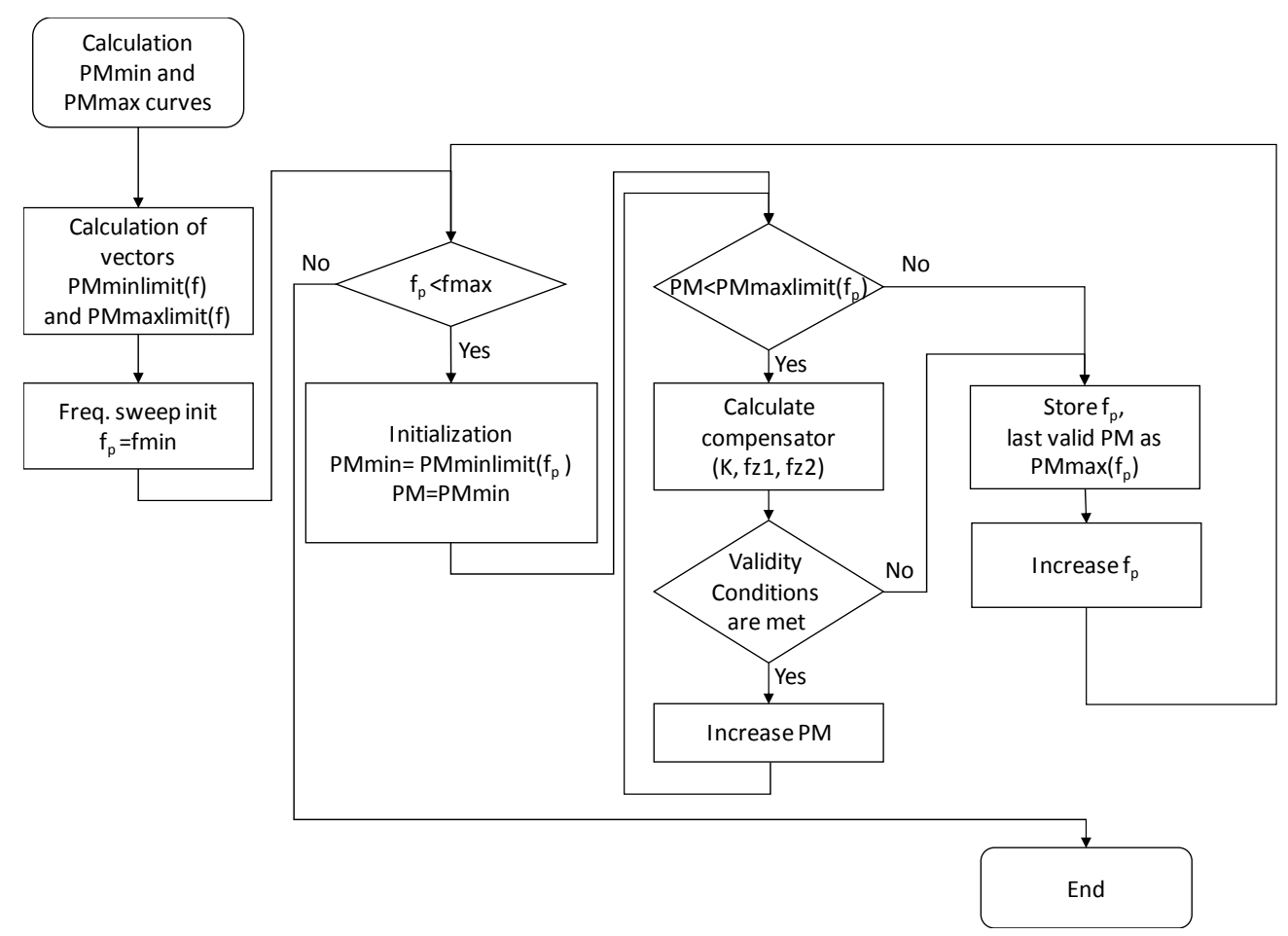

Figure 7. Flowchart of the procedure to calculate the limits of the performance space $\left(f_{c}, P M\right)$.

The flowchart in Figure 7 describes the procedure to calculate the limits of the performance space. Moreover, it can be also used to plot the complete performance space $\left(f_{c}, P M\right)$ if PMminlimit(f) and PMmaxlimit(f) are set to the minimum and maximum PM values, respectively, and the validity conditions do not interrupt the loop but are used to classify the solutions.

The most valuable contribution of this algorithm is the ability to analyze all possible designs very quickly and compare different compensator types or the influence of design parameters. The following discussion illustrates this added value.

Limit cycling conditions are analyzed in Figure 8 in the case of PID compensators. Different types of compensators and design parameters (ratio of zero frequency, $K_{1}$, and $K_{2}$ ) are considered by running the algorithm of Figure 7 once per compensator type and design parameter $K_{1}$ or $K_{2}$. Note that limit cycling conditions, as described in Section 2.4 , can be easily included in the calculations.

The area limited by the red line in Figure 8 corresponds to the pairs $\left(f_{c}, P M\right)$ that do not meet the integral gain limit cycling condition. This area is smaller, as the separation between the zero frequencies increases (ratio $f_{z 2} / f_{z 1}$ for PID2 and ratio $f_{z 2} / f_{c}$ for PID1 decrease). The area limited by the green line in the plots of Figure 8 is the set of solutions that do not meet the limit cycling condition referring to the gain margin. This condition is more related to the frequency itself than to the type of compensator or the value of its design parameter. No significant gains are obtained when the frequencies of the zeros are very different.

Merged results for the PID1 and PID2 compensators are shown in Figure 9. Areas limited by red or green lines in Figure 8 are now discarded designs due to the existence of limit cycles. The area limited by the blue line is the performance space free of limit cycling. In the case of PID2, there is a remarkable difference when the frequency of the zeros changes: the lower the ratio $f_{z 2} / f_{z 1}$, the wider the allowable performance space. The integral gain limit cycling condition has, in this case, the strongest influence on the allowable designs. PID1 exhibits similar behavior, although the differences are slightly less significant. Both PID1 and PID2 provide similar results when the ratios $f_{z 2} / f_{z 1}$ or 
$f_{z 2} / f_{c}$ are very small ( 0.01 in this case). This fact could be used as a criterion to minimize the possibility of a limit cycle, using a high separation between the frequencies of the zeros (low values of $f_{z 2} / f_{z 1}$, and $f_{z 2} / f_{c}$ ). However, the response of the system at low frequencies can be degraded, as depicted in Section 2.5 and as it is confirmed in the experimental measurements. A trade-off should be achieved between the reduction of the area with limit cycling due to integral gain and the degradation of the low-frequency response.
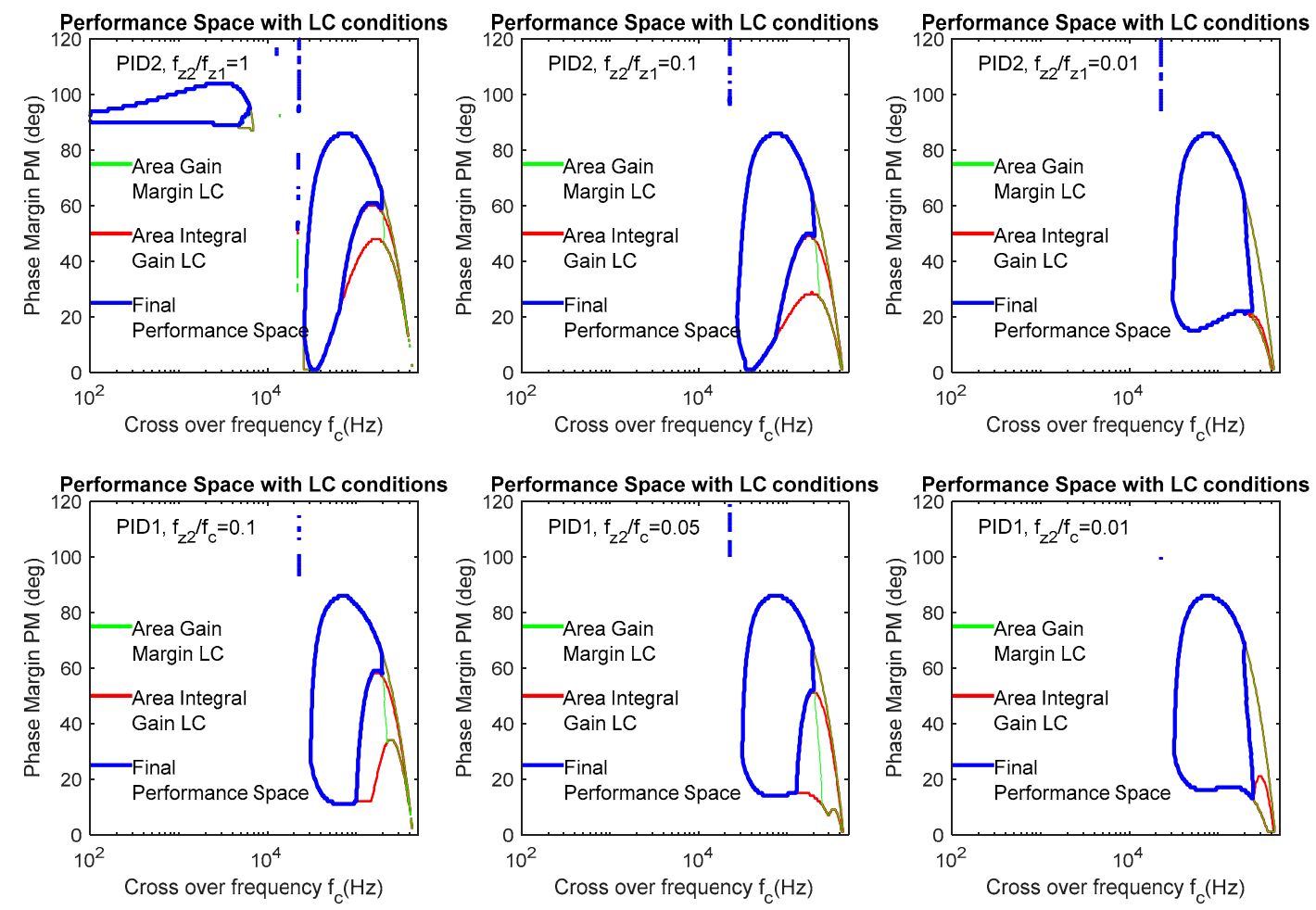

Figure 8. Performance space for PID compensators, remarking areas discarded due to the limit cycling (LC) conditions.

Figure 10 illustrates the limit cycling. Time-domain simulations with a PSIM simulator have been carried out. The ADC and the DPWM (digital PWM) are quantized, but the DPWM has a much higher resolution than the ADC. Therefore, limit cycling concerning the relative resolution of ADC and DPWM [27] is avoided. Small steps in the output voltage reference are introduced to force transient intervals. After those periods, the system enters into a steady state. If there are still oscillations in the output voltage, there is a limit cycle. As predicted by the performance space of Figure 9, two of the six analyzed designs (PID2 $f_{z 2} / f_{z 1}=1$ and PID1 $f_{z 2} / f_{c}=0.1$ ) are outside the performance space in the area corresponding to limit cycling conditions. The other designs do not suffer from limit cycling, agreeing with Figure 9. Limit cycling failures occur in the case of the lower zero-frequency separation, confirming the trends identified in the previous paragraphs.

A final performance space has been calculated and plotted in Figure 11. This plot summarizes the best possible design for every dynamic requirement $\left(f_{c}, P M\right)$. Note that all considered compensator topologies cover some area in the performance space.

The first criterion to select the best solution is simplicity: PI is preferred to PID because of the reduced number of coefficients, Equations (12), (15) and (18). The second criterion is the value of index $L$, as defined in Section 2.5-that is, in the case of PID compensators achieving a feasible design for a given specified $\left(f_{c}, P M\right)$, the preferred solution is the one with the lower index $L$. 


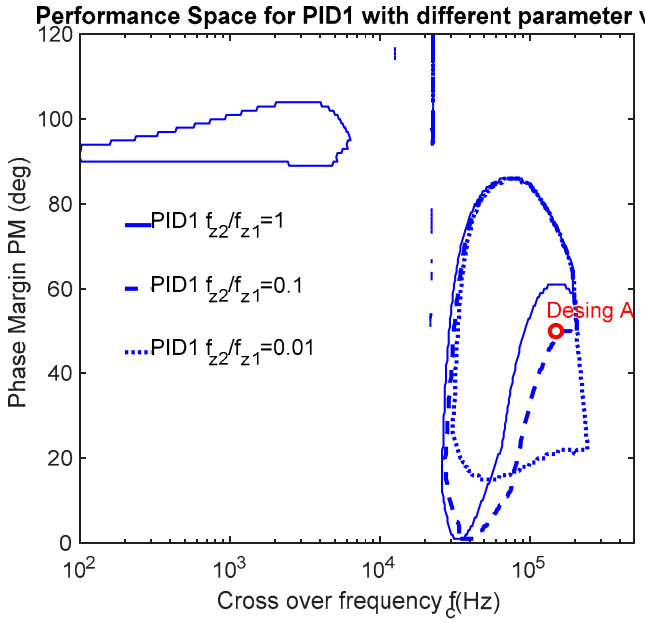

(a)

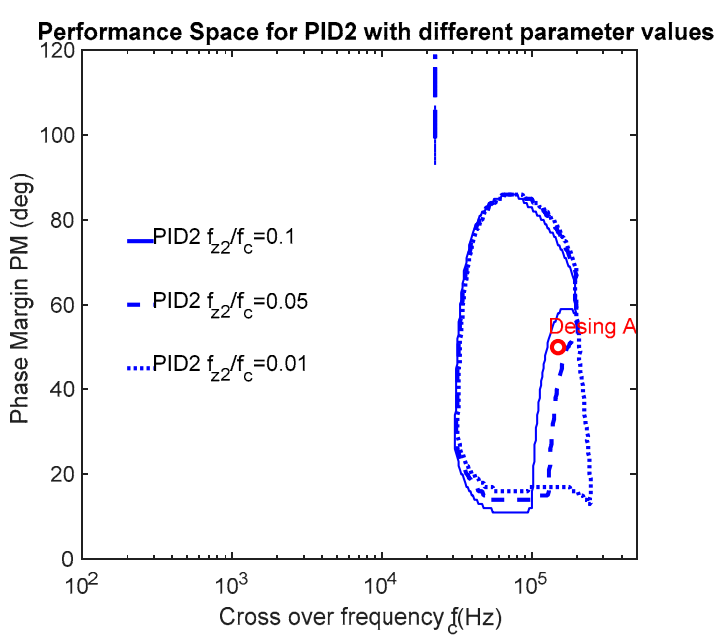

(b)

Figure 9. Performance space for the two different PID compensators, considering different values of the design parameter for each of them: (a) PID1; (b) PID2.
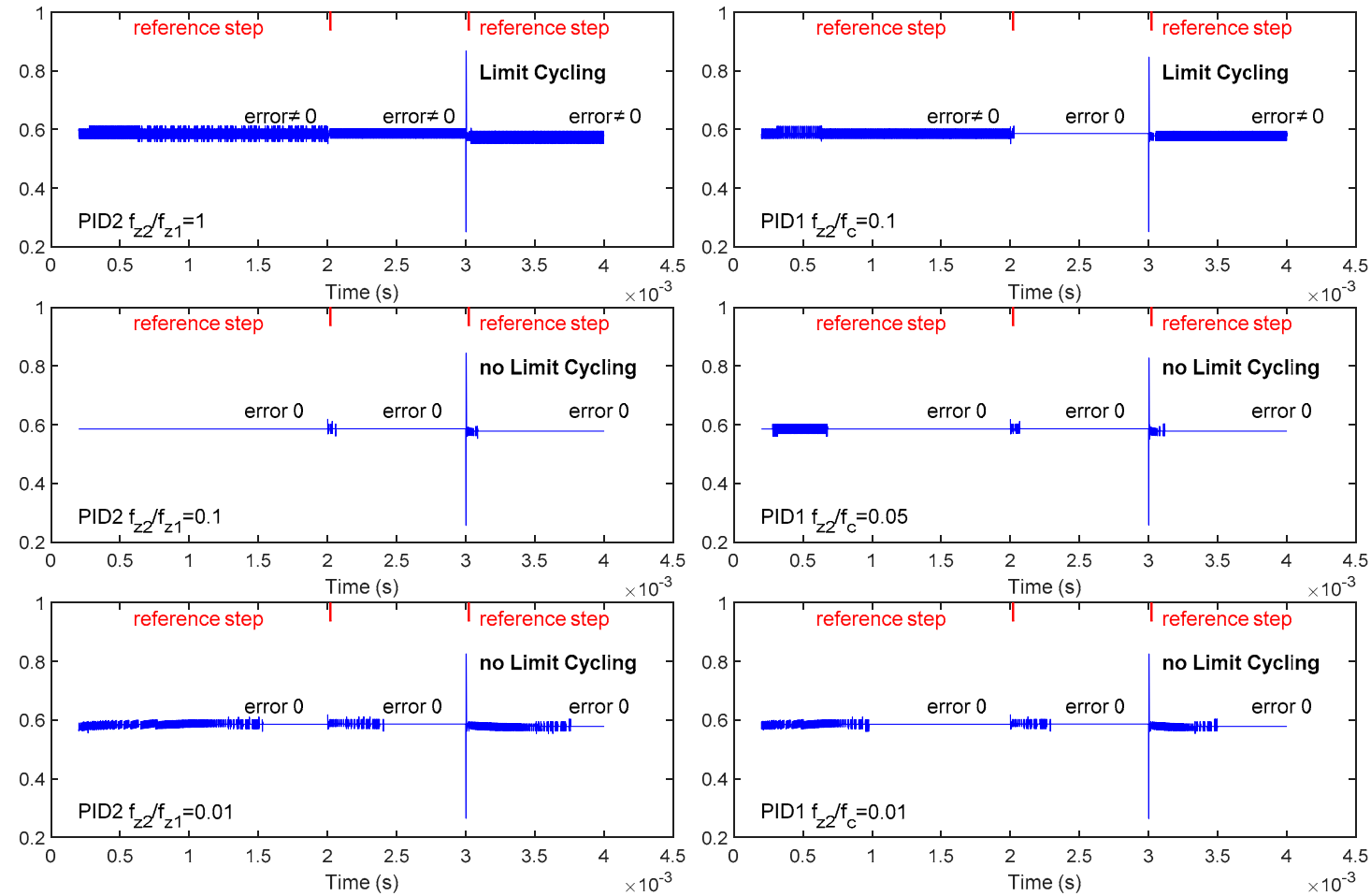

Figure 10. Time response to reference steps for different compensators corresponding to design A (Figure 8). A limit cycle exists for PID $1 f_{z 2} / f_{z 1}=1$ and PID2 $f_{z 2} / f_{c}=0.1$, as predicted by the performance space.

Analyzing this example, the PI is obviously the best solution for a low-frequency requirement. Even if the PID can provide low values of $f_{c}$, the PI has a simpler implementation that implies less calculation in the difference equation. Designs using the PI compensator beyond the resonance frequency of the plant are limited to low values of $P M$, which in practice have no significant interest.

The preferred option for values of $f_{c}$ beyond the resonance frequency is the PID2 with two zeros at the same frequency. The limitation due to the integral gain limit cycling condition can be overcome using either PID2 or PID1, with higher separation between zero frequencies. 


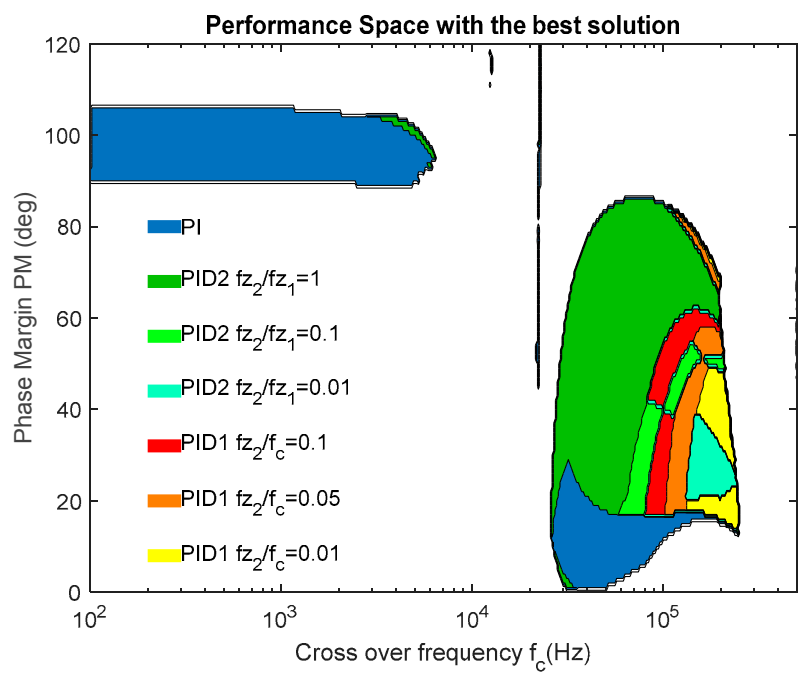

Figure 11. Performance space considering seven different compensators and selecting the best possible solution among them for each point of the performance space $\left(f_{c}, P M\right)$.

All results presented in this section have been obtained by implementing in Matlab the algorithm to generate the performance space (Figure 7). Apart from the comparison of different compensators, the same procedure can be used to perform a sensitivity analysis of other parameters, such as time delays and sampling frequency [35].

\section{Experimental Validation}

An actual prototype has been built and tested in the laboratory to validate the proposed design procedure, the models, and the assumptions considered in this work. The digital controller has been implemented in a Zybo board, including a Xilinx Zynq-7010 device. An external ADC was used (ADS7476A), limiting the resolution to 10 bits. The specification of the power converter is as follows: input voltage $\mathrm{Vin}=8 \mathrm{~V}$, output voltage $\mathrm{Vo}=4 \mathrm{~V}$; output inductance $\mathrm{L}_{\mathrm{O}}=76 \times 10^{-6} \mathrm{H}$; output capacitance $C_{O}=100 \times 10^{-6} \mathrm{~F}$; load resistance Ro $=10 \Omega$; switching frequency fsw $=100 \times 10^{3} \mathrm{~Hz}$. The transfer function of the power converter $G_{v d}$ (Figure 1) has not been calculated, but was measured with a frequency response analyzer. Therefore, all parasitic components (inductor and capacitor series resistances, MOSFET conduction resistance, etc.) are considered in the compensator calculation. The uncompensated loop gain $T_{u}$ is shown in Figure 12, as well as the performance space, without considering the limit cycling conditions. There are some differences with the performance space presented in Figure $6 \mathrm{~b}$ due to the differences in the uncompensated open-loop transfer function:

- The total time delay produces a significant phase loss beyond the resonance frequency of the uncompensated open loon gain, limiting the feasible solutions at high frequency (PMmax limit). This phase loss is compensated partially by the effect of the equivalent series resistance of the output capacitor;

- The $\mathrm{Q}$ factor is lower, and so in the performance space, there are possible solutions at crossover frequencies close to the resonance frequency.

The combination of the performance spaces for the different type of compensators and different design parameters is shown in Figure 13. In this case, PI ad PID2 with $f_{z 2} / f_{z 1}=1$ are the compensator topologies that cover the major part of the area of possible designs. The PID1 topology provides possible designs at high $f_{c}$ and low $P M$, where the PID2 with $f_{z 2} / f_{z 1}=1$ does not meet the limit cycling condition regarding the integral gain.

The resulting open-loop gain has been measured in the actual prototype for three dynamic specifications, named as design point 1, 2, and 3, detailed in Figure 13. For each design point, both PID1 and PID2 have been designed with $f_{z 2} / f_{c}=0.1$ and $f_{z 2} / f_{z 1}=1$, respectively. Measurements 
and theoretical predictions are plotted in Figure 14, which match very well, especially near the crossover frequency. Another interesting comparison between PID1 and PID2 is the magnitude of the open-loop gain for the same design point. Bellow the cross-frequency in each case, PID2 reaches a higher magnitude than PID1, which means a lower L index. That is why, in the performance space plotted in Figure 13, PID2 is a better solution than PID1 for the three illustrated design points. This result is also illustrated in the time-domain waveforms appearing in Figure 15. The output voltage has been measured when a voltage reference step is applied. In the left plot, the six different possibilities for design-point 3 are shown. Although the same crossover frequency and phase margin are selected for the compared waveforms, the system exhibits a different time-response due to the zero location, as shown in previous sections. PID2 with $f_{z 2} / f_{z 1}=1$ is the fastest response, matching with the choice to plot the performance space in Figure 13. In the right side of Figure 15, the time-response for design-point 1 for a single compensator is shown to illustrate that, despite the high cross-over frequency, no limit cycles are produced after the transitions, as predicted by the theory.

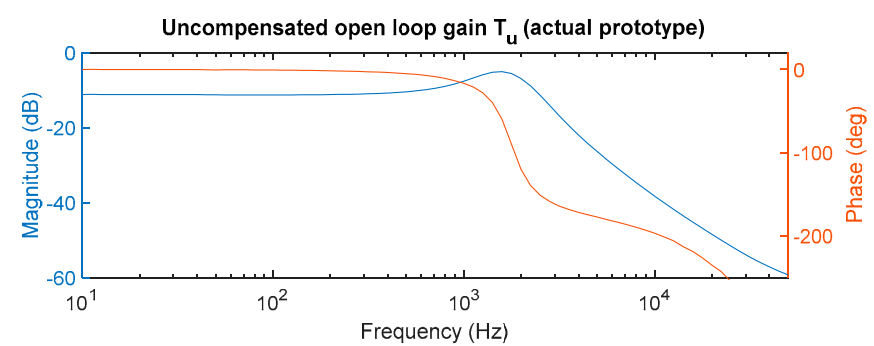

(a)

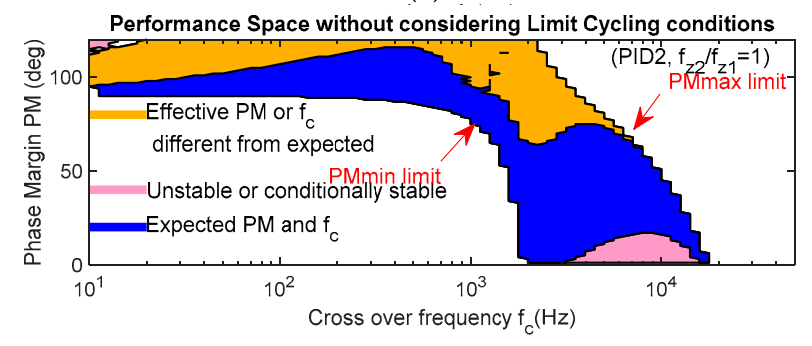

(b)

Figure 12. (a) The actual prototype's uncompensated open loop gain; (b) performance space without considering limit cycling conditions.

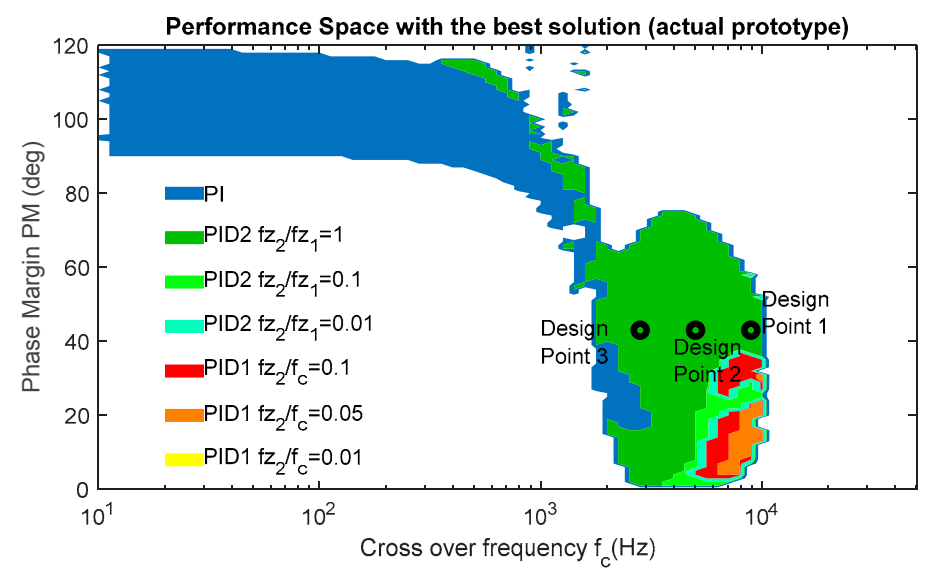

Figure 13. Performance space for the actual prototype with the best solution for each point. 

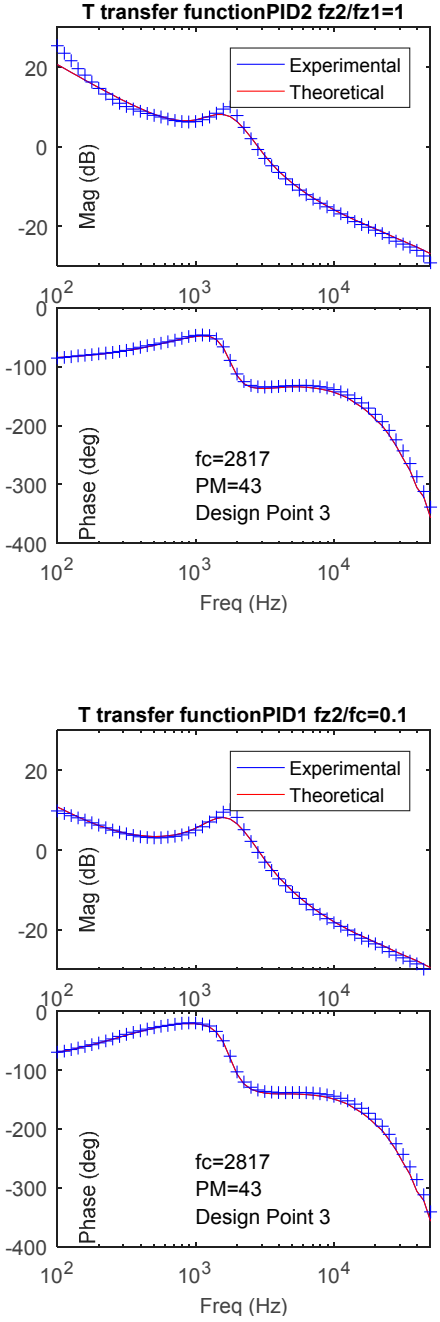
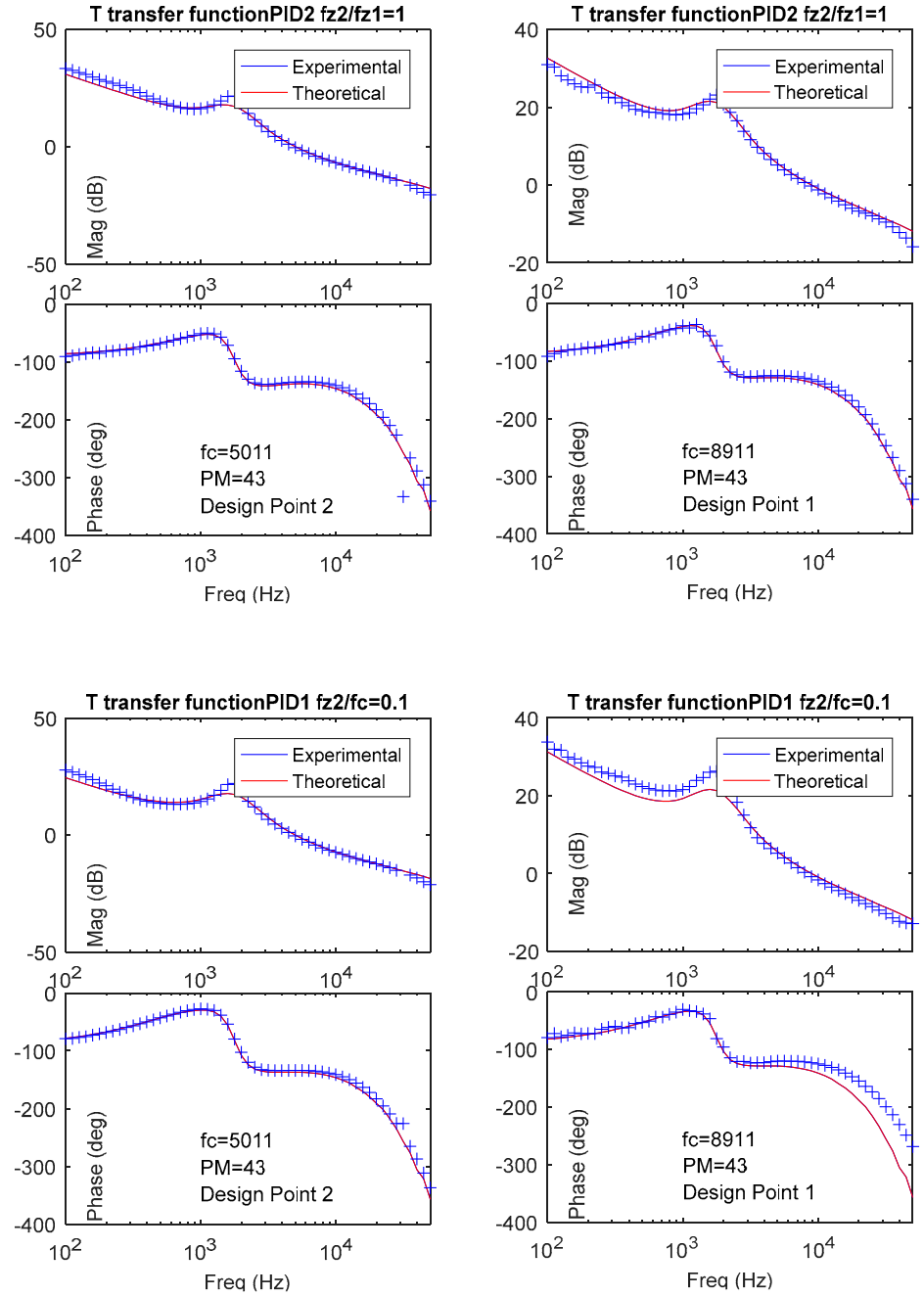

Figure 14. Comparison between the experimental measurements and the theoretical predictions for different PID compensators, considering the three design points in Figure 13.

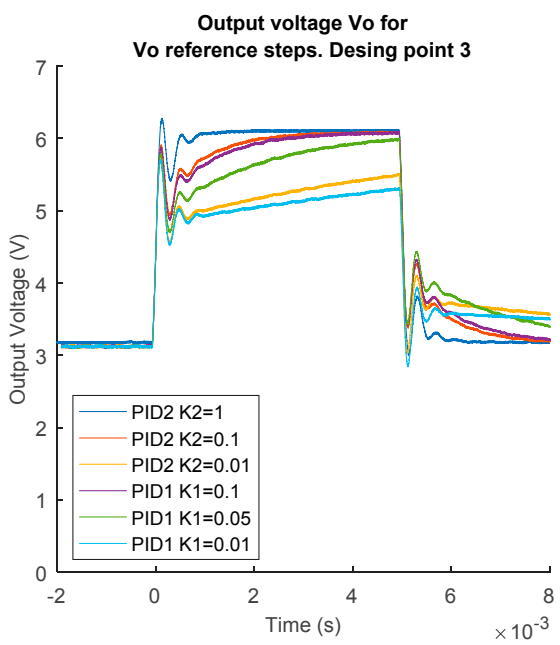

(a)

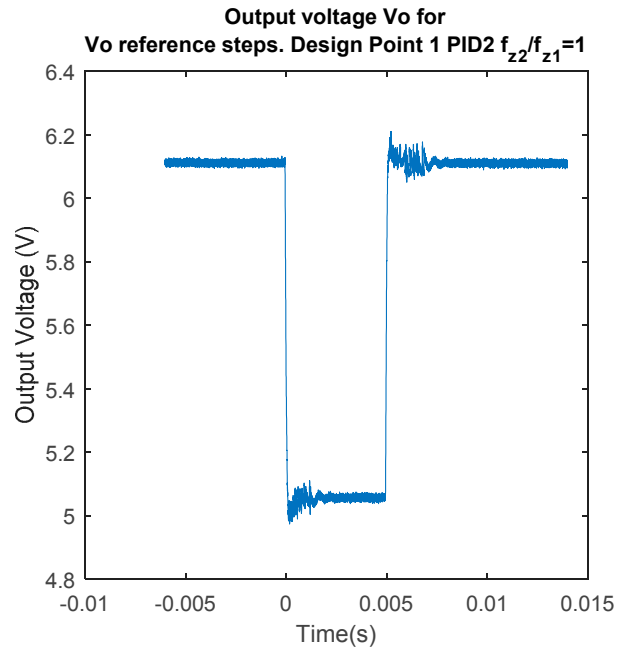

(b)

Figure 15. Experimental time-domain waveforms when there is a step in the voltage reference. (a) Step response for different designs with $f_{c}=2817 \mathrm{~Hz}, \mathrm{PM}=43^{\circ}$ (Design point 3); (b) step response for design-point 1 , using 9 bits in the ADC (note that there is no limit cycling). 


\section{Conclusions}

A frequency-based design approach for the calculation of a digital compensator for DC/DC power-switching converters has been presented. It is based on the analytical calculation of the coefficients of the compensator from the dynamic specifications phase margin, $P M$, and cross-over frequency, $f_{c}$, considering the sampling frequency, delay effects, and limit cycling conditions. The approach is focused on the identification of the dynamic specifications that in fact can be achieved, and its graphical representation on a $\left(f_{c}, P M\right)$ axis. Designs that are not feasible, do not fulfill the requirements, are unstable, or do not meet the limit cycling conditions, are excluded from the performance space.

The design procedure has been automatized. A simple algorithm can generate all feasible designs and plot the performance space. It allows for the identification of the dynamic limitations of a given power converter with a given compensator topology. The most important benefit provided by this approach is the ability to perform a quick and straightforward comparison of compensator topologies or sensitivity analysis of a specific parameter, ensuring the feasibility of the proposed designs.

Three compensator topologies have been considered in the calculations: PI and PID, with two different criteria to establish the frequency of the two zeros (PID1 and PID2). The design procedure has been applied in a particular example, considering seven different compensator types and choosing the more suitable design for each point $\left(f_{c}, P M\right)$ using a proposed figure of merit calculated from the frequency response.

The described analysis based on the performance space also allows for determination of the simplest compensator design in terms of the dynamic performance, the complexity of the implementation, and the computational resources. In the analyzed example, PI is the best option for low-frequency dynamic requirements. In general, the option covering a larger area in the performance space is PID with two zeros at the same frequency. However, this performance space can be limited by the integral limit cycling condition. Other PID designs with separated zeros can overcome this limitation, but the time response should be assessed. The experimental results of a lab prototype agree with the theoretical predictions.

Author Contributions: Conceptualization, P.Z., C.F., A.L. and A.B.; Data curation, M.A.G.; Funding acquisition, C.F. and A.B.; Methodology, P.Z. and C.F.; Software, M.A.G.; Validation, M.A.G.; Writing-original draft, P.Z. and C.F.; Writing-review \& editing, M.A.G., A.L. and A.B.

Funding: This work has been funded by the Ministerio de Economía, Comercio y Competitividad, Gobierno de España and ERDF funds, through projects DPI2017-88062-R, “Electrónica de Potencia Integrada e Inteligente Para el Control y la Gestión de la Energía en la IIOT" EPIIOT and project DPI2014-53685-C2-1-R, "Storage and Energy Management for Hybrid Electric Vehicles based on Fuel Cell, Battery and Supercapacitors"-ELECTRICAR-AG.

Conflicts of Interest: The authors declare no conflict of interest.

\section{References}

1. Sathishkumar, P.; Krishna, T.N.; Khan, M.A.; Zeb, K.; Kim, H.J. Digital Soft Start Implementation for Minimizing Start Up Transients in High Power DAB-IBDC Converter. Energies 2018, 11, 956. [CrossRef]

2. Costabeber, A.; Mattavelli, P.; Saggini, S. Digital Time-Optimal Phase Shedding in Multiphase Buck Converters. IEEE Trans. Power Electron. 2010, 25, 2242-2247. [CrossRef]

3. Rodríguez, J.; Lamar, G.D.; Aller, G.D.; Miaja, F.P.; Sebastián, J. Efficient Visible Light Communication Transmitters Based on Switching-Mode dc-dc Converters. Sensors 2018, 18, 1127. [CrossRef] [PubMed]

4. Shirazi, M.; Morroni, J.; Dolgov, A.; Zane, R.; Maksimovic, D. Integration of Frequency Response Measurement Capabilities in Digital Controllers for DC/DC Converters. IEEE Trans. Power Electron. 2008, 23, 2524-2535. [CrossRef]

5. Shirazi, M.; Zane, R.; Maksimovic, D. An Autotuning Digital Controller for DC/DC Power Converters Based on Online Frequency-Response Measurement. IEEE Trans. Power Electron. 2009, 24, 2578-2588. [CrossRef]

6. Stefanutti, W.; Mattavelli, P.; Saggini, S.; Ghioni, M. Autotuning of Digitally Controlled DC-DC Converters Based on Relay Feedback. IEEE Trans. Power Electron. 2007, 22, 199-207. [CrossRef] 
7. Costabeber, A.; Mattavelli, P.; Saggini, S.; Bianco, A. Digital Autotuning of DC-DC Converters Based on a Model Reference Impulse Response. IEEE Trans. Power Electron. 2011, 26, 2915-2924. [CrossRef]

8. Stefanutti, W.; Saggini, S.; Mattavelli, P.; Ghioni, M. Power Line Communication in Digitally Controlled DC-DC Converters Using Switching Frequency Modulation. IEEE Trans. Ind. Electron. 2008, 55, 1509-1518. [CrossRef]

9. Park, Y.J.; Khan, Z.H.; Oh, S.J.; Jang, B.G.; Ahmad, N.; Khan, D.; Abbasizadeh, H.; Shah, S.A.; Pu, Y.G.; Hwang, K.C.; et al. Single Inductor-Multiple Output DPWM DC-DC Boost Converter with a High Efficiency and Small Area. Energies 2018, 11, 725. [CrossRef]

10. Xie, K.; Hu, G.; Yi, H.; Lyu, Z.; Xiang, Y. A Novel Digital Control Method of a Single-Phase Grid-Connected Inverter Based on a Virtual Closed-Loop Circuit and Complex Vector Representation. Energies 2017, 10, 2068. [CrossRef]

11. Thi Kim Nga, T.; Park, S.M.; Park, Y.J.; Park, S.H.; Kim, S.; Van Cong Thuong, T.; Lee, M.; Hwang, K.; Yang, Y.; Lee, K.Y. A Wide Input Range Buck-Boost DC-DC Converter Using Hysteresis Triple-Mode Control Technique with Peak Efficiency of 94.8\% for RF Energy Harvesting Applications. Energies 2018, 11, 1618. [CrossRef]

12. Jackson, D.K.; Leeb, S.B. A digitally controlled amplifier with ripple cancellation. IEEE Trans. Power Electron. 2003, 18, 486-494. [CrossRef]

13. Bae, H.-S.; Yang, J.-H.; Lee, J.-H.; Cho, B.-H. Digital state feedback current control using the pole placement technique. J. POWER Electron. 2007, 7, 213-221.

14. Bae, H.S.; Yang, J.H.; Lee, J.H.; Cho, B.H. Digital state feedback control and feed-forward compensation for a parallel module DC-DC converter using the pole placement technique. In Proceedings of the IEEE Applied Power Electronics Conference and Exposition-APEC, Austin, TX, USA, 24-28 February 2008; pp. 1722-1727.

15. Peretz, M.M.; Ben-Yaakov, S. Time-Domain Design of Digital Compensators for PWM DC-DC Converters. IEEE Trans. Power Electron. 2012, 27, 284-293. [CrossRef]

16. Miao, B.; Zane, R.; Maksimović, D. Automated digital controller design for switching converters. In Proceedings of the PESC Rec. IEEE Annual Power Electronics Specialist Conference, Recife, Brazil, 12-16 June 2005; pp. 2729-2735.

17. Chui, M.Y.; Ki, W.-H.; Tsui, C.-Y. A programmable integrated digital controller for switching converters with dual-band switching and complex pole-zero compensation. IEEE J. Solid-State Circuits 2005, 40, 772-780. [CrossRef]

18. Garcia, O.; de Castro, A.; Soto, A.; Oliver, J.A.; Cobos, J.A.; Cezon, J. Digital control for power supply of a transmitter with variable reference. In Proceedings of the Twenty-First Annual IEEE Applied Power Electronics Conference and Exposition, Dallas, TX, USA, 19-23 March 2006; p. 6.

19. Erickson, R.W.; Maksimovic, D. Fundamental of Power Electronics, 2nd ed.; Springer: Berlin, Germany, 2001.

20. Venable, $\mathrm{H}$. The $\mathrm{K}$ factor: A new mathematical tool for stability analysis and synthesis. In Proceedings of the Powercon 10, San Diego, CA, USA, 22-24 March 1983; pp. 1-10.

21. Corradini, L.; Mattavelli, P.; Stefanutti, W.; Saggini, S. Simplified Model Reference-Based Autotuningfor Digitally Controlled SMPS. IEEE Trans. Power Electron. 2008, 23, 1956-1963. [CrossRef]

22. Yang, C.; Liu, C.; Tsai, C. Direct digital compensator design for switching converters. In Proceedings of the 2010 International Symposium on Next Generation Electronics, Kaohsiung, Taiwan, 18-19 November 2010; pp. 143-146.

23. Bjeletić, A.; Corradini, L.; Maksimović, D.; Zane, R. Specifications-driven design space boundaries for point-of-load converters. In Proceedings of the IEEE Applied Power Electronics Conference and Exposition-APEC, Fort Worth, TX, USA, 6-11 March 2011; pp. 1166-1173.

24. Martinez, C.; Valdivia, V.; Roldan, A.M.; Lourido, J.; Quesada, I.; Lucena, C.; Zumel, P.; Barrado, A. Efficient CAD tool for power electronics compensator design. In Proceedings of the 2010 IEEE Energy Conversion Congress and Exposition, Atlanta, GA, USA, 12-16 September 2010.

25. Fernández, C.; Lázaro, A.; Zumel, P.; Valdivia, V.; Martínez, C.; Barrado, A. Design space boundaries of linear compensators applying the k-factor method. In Proceedings of the 2013 Twenty-Eighth Annual IEEE Applied Power Electronics Conference and Exposition (APEC), Long Beach, CA, USA, 17-21 March 2013; pp. 2706-2711.

26. Peterchev, A.V.; Sanders, S.R. Quantization resolution and limit cycling in digitally controlled PWM converters. IEEE Trans. Power Electron. 2003, 18, 301-308. [CrossRef] 
27. Corradini, L.; Maksimovic, D.; Mattavelli, P.; Zane, R. Amplitude Quantization. In Digital Control of High-Frequency Switched-Mode Power Converters; Wiley-IEEE Press: Piscataway, NJ, USA, 2015; p. 360.

28. Lin, Y.C.; Chen, D.; Wang, Y.T.; Chang, W.H. A Novel Loop Gain-Adjusting Application Using LSB Tuning for Digitally Controlled DC-DC Power Converters. IEEE Trans. Ind. Electron. 2012, 59, 904-911. [CrossRef]

29. Al-Atrash, H.; Batarseh, I. Digital controller design for a practicing power electronics engineer. In Proceedings of the APEC 07-Twenty-Second Annual IEEE Applied Power Electronics Conference and Exposition, Anaheim, CA, USA, 25 February-1 March 2007; pp. 34-41.

30. Corradini, L.; Maksimovic, D.; Mattavelli, P.; Zane, R. Discrete-Time Modeling. In Digital Control of High-Frequency Switched-Mode Power Converters; Wiley-IEEE Press: Piscataway, NJ, USA, 2015; p. 360.

31. Maksimovic, D.; Zane, R. Small-Signal Discrete-Time Modeling of Digitally Controlled PWM Converters. IEEE Trans. Power Electron. 2007, 22, 2552-2556. [CrossRef]

32. VandeSype, D.M.; DeGusseme, K.; DeBelie, F.M.L.L.; VandenBossche, A.P.; Melkebeek, J.A. Small-Signal z-Domain Analysis of Digitally Controlled Converters. IEEE Trans. Power Electron. 2006, 21, 470-478. [CrossRef]

33. Corradini, L.; Maksimovic, D.; Mattavelli, P.; Zane, R. Digital Control. In Digital Control of High-Frequency Switched-Mode Power Converters; Wiley-IEEE Press: Piscataway, NJ, USA, 2015; p. 360.

34. Duarte-Mermoud, M.A.; Prieto, R.A. Performance index for quality response of dynamical systems. ISA Trans. 2004, 43, 133-151. [CrossRef]

35. Zumel, P.; Fernandez, C.; Granda, M.A.; Lazaro, A.; Sanz, M.; Barrado, A. Simple method of direct digital design of compensator for DC-DC converters. In Proceedings of the 2016 IEEE 17th Workshop on Control and Modeling for Power Electronics, COMPEL 2016, Trondheim, Norway, 27-30 June 2016.

(C) 2018 by the authors. Licensee MDPI, Basel, Switzerland. This article is an open access article distributed under the terms and conditions of the Creative Commons Attribution (CC BY) license (http:/ / creativecommons.org/licenses/by/4.0/). 